Case

CrossMark \& click for updates

\section{Prominent Cognitive Dysfunction without Motor Impairment Following Anterior Choroidal Artery Infarction: a Case Report}

\author{
Tae-ha Park, Jinyoung Park, Yoon Ghil Park, Seo Yeon Yoon
}

\section{Highlights}

Revised: Aug 24, 2016

Accepted: Sep 6, 2016

\section{Correspondence to} Yoon Ghil Park

Department of Rehabilitation Medicine and

Rehabilitation Institute of Neuromuscular

Disease, Gangnam Severance Hospital, Yonsei

University College of Medicine, 211 Eonju-ro,

Gangnam-gu, Seoul 06273, Korea.

Tel: +82-2-2019-3492

Fax: +82-2-3463-7585

E-mail:drtlc@yuhs.ac
- Neuropsychological and perceptual deficits are uncommon by AChAI.

- Infarction in internal capsule supplied by the AChA can deteriorate cognition.

- Further functional brain imaging would be helpful to reveal the connectivity. 


\section{Case}

CrossMark \& click for updates
a

\section{OPEN ACCESS}

Received: Jul 11, 2016

Revised: Aug 24, 2016

Accepted: Sep 6, 2016

\section{Correspondence to}

Yoon Ghil Park

Department of Rehabilitation Medicine and

Rehabilitation Institute of Neuromuscular

Disease, Gangnam Severance Hospital, Yonsei

University College of Medicine, 211 Eonju-ro,

Gangnam-gu, Seoul 06273, Korea.

Tel: +82-2-2019-3492

Fax: +82-2-3463-7585

E-mail: drtlc@yuhs.ac

Copyright (c) 2016 Korean Society for Neurorehabilitation

This is an Open Access article distributed under the terms of the Creative Commons Attribution Non-Commercial License (http:// creativecommons.org/licenses/by-nc/4.0/) which permits unrestricted non-commercial use, distribution, and reproduction in any medium, provided the original work is properly cited.

Conflict of Interest

The authors have no potential conflicts of interest to disclose.

\section{Prominent Cognitive Dysfunction without Motor Impairment Following Anterior Choroidal Artery Infarction: a Case Report}

\author{
Tae-ha Park,' Jinyoung Park, 'Yoon Ghil Park,' Seo Yeon Yoon' \\ 'Department of Rehabilitation Medicine and Rehabilitation Institute of Neuromuscular Disease, Yonsei \\ University College of Medicine, Seoul, Korea \\ ${ }^{2}$ Department of Rehabilitation Medicine, Bundang Jesaeng General Hospital, Seongnam, Korea
}

\begin{abstract}
Neurological deficits commonly associated with anterior choroidal artery infarction (AChAI) include hemiplegia, hemisensory loss, and homonymous hemianopsia, while neuropsychological and perceptual deficits are uncommon. Prominent cognitive function impairment has rarely been reported. Here, we report a case of AChAI with prominent cognitive function impairment without motor deterioration. In contrast to the typical clinical features of AChAI, near complete and rapid motor recovery was observed, while cognitive impairment persisted despite rehabilitation therapy.
\end{abstract}

Keywords: Anterior Choroidal Artery Infarction; Cognitive Impairment; Internal Capsule

\section{INTRODUCTION}

The anterior choroidal artery (AChA) originates from the internal carotid artery and distal to the posterior communicating artery (PCoA). The AChA supplies crucial motor and sensory structures, such as the posterior limb of the internal capsule, the cerebral peduncle, the choroid plexus, and the optic tract [1].

Neurological deficits commonly associated with anterior choroidal artery infarction (AChAI) include hemiplegia, hemisensory loss, and homonymous hemianopsia. However, prominent cognitive dysfunction with relatively less prominent motor impairment has rarely been reported. We experienced a case of AChAI with prominent cognitive impairment and relatively less prominent motor function impairment. In contrast to the typical clinical features of AChAI, near complete and rapid motor recovery occurred, while cognitive impairment persisted despite rehabilitation therapy. 


\section{CASE REPORT}

A 59-year-old right-handed woman was hospitalized with acute-onset severe headache. She had received treatment for hypertension but did not have any history of serious illness, neuropsychological disorders and family history. Brain magnetic resonance imaging (MRI), magnetic resonance angiography (MRA), and cerebral angiogram revealed an unruptured aneurysms of the anterior communicating artery (ACoA), left distal internal carotid artery and AChA. Following twice successful clipping surgery, abruptly right-sided weakness and cognitive impairment developed. The patient's vital signs were stable, and laboratory results were within normal limits. Acute infarction in the posterior limb of the left internal capsule was diagnosed using diffusion weighted brain imaging (DWI), and there were no other infarction sites (Fig. 1). The left AChA was not visualized on angiogram (Fig. 2).

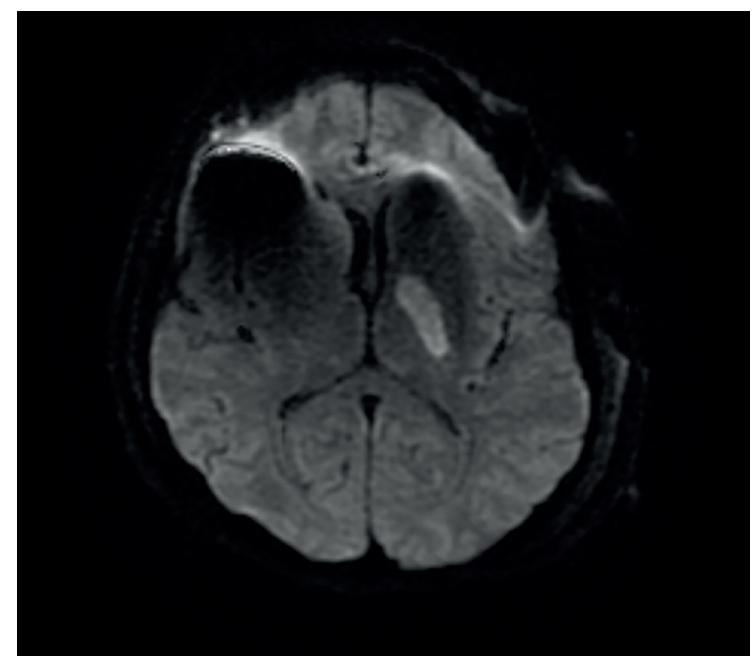

Fig. 1. Diffusion-weighted image of brain MRI.

Acute infarction in the posterior limb of the left internal capsule was revealed on diffusion-weighted image of brain MRI.
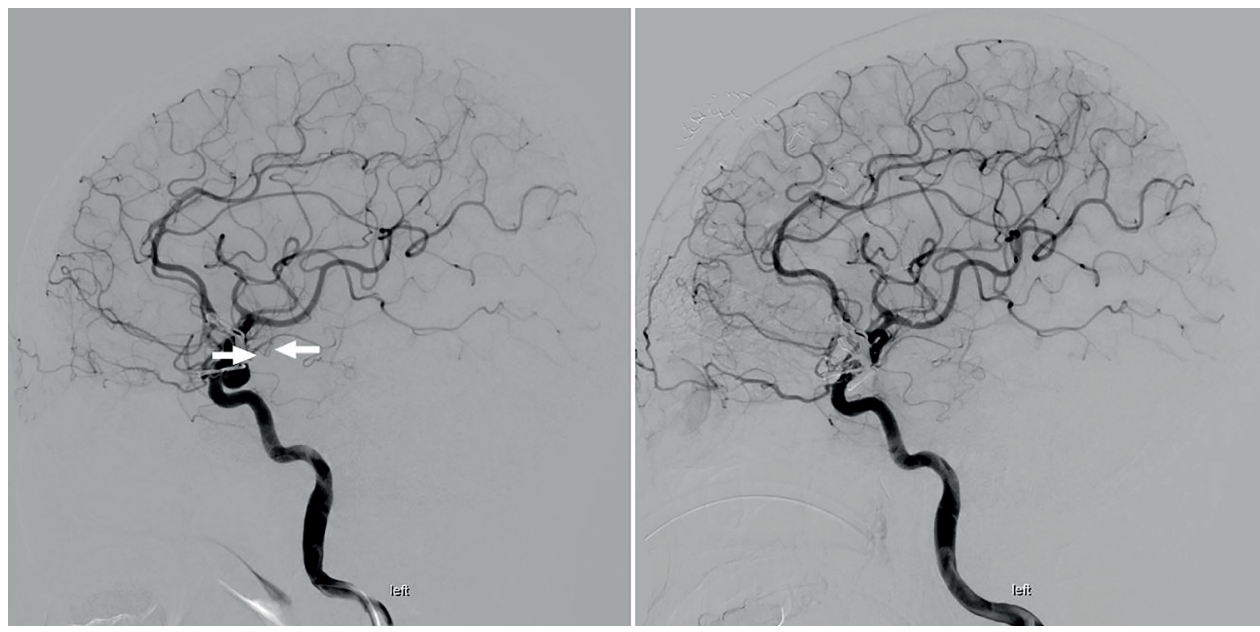

Fig. 2. Cerebral angiography before and after cerebral infarction.

While the left anterior choroidal artery was well visualized in angiography taken before cerebral infarction (left image, white arrows), the artery is not visualized on the angiography taken after cerebral infarction (right image). 
At the time of diagnosis, both motor weakness and cognitive impairment were prominent. Right-sided upper and lower extremities were graded trace according to the manual muscle test (MMT). The patient was mentally alert, but the Korean Mini-Mental State Examination (K-MMSE) could not be performed because of severe cognitive impairment and aphasia. She was entirely dependent on others for activities of daily living (ADL).

Twenty days after stroke, motor weakness recovered noticeably. Motor grades of the right upper and lower extremities were fair minus and poor, respectively. The patient needed maximal assistance when performing activities that involved cognitive function. Her Functional Independence Measure (FIM) score was 42, but K-MMSE parameters still could not be evaluated. The patient showed clinical signs of global aphasia with an Aphasia Quotient (AQ) score of 26.6. An Intelligence Quotient (IQ) below 60 indicated a mild to moderate level of mental retardation. The patient's Social Quotient (SQ) was 2, equivalent to a social age of 1 year. Diffusion tensor imaging (DTI) brain tractography revealed that the corticospinal tract was intact (Fig. 3).

After 37 days from stroke, right-side motor grading improved to 'good' in both the upper and lower extremities. Cognitive function was slightly improved and a FIM score increased to 91, although cognitive impairment was still prominent. The patient's K-MMSE score was 15 , and recovery was predominantly shown in the orientation and language field. IQ improved to 65, indicating mild mental retardation. The patient also showed recovery from global to anomic aphasia, an AQ score of 73.6. Tables 1 and 2 show the patient's hospital course.

After discharge, the patient received cognitive rehabilitation therapy consistently. About 18 months after infarction onset, K-MMSE score improved to 25 and the patient could perform all ADLs with supervision. Despite remaining cognitive deficits, the patient was able to return to work.

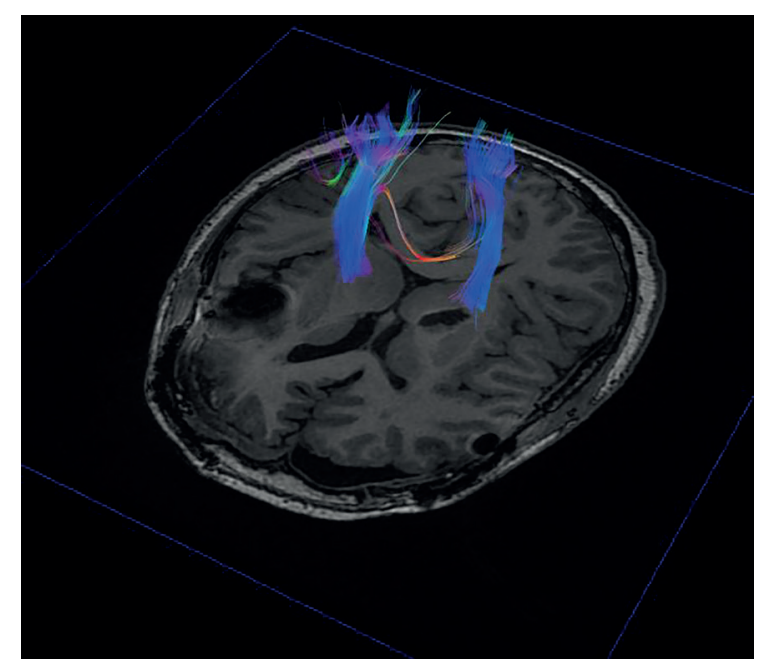

Fig. 3. Brain tractography by diffusion tensor image.

The tractography by diffusion tensor image showed bilaterally intact corticospinal tracts. 
Table 1. K-MMSE scores

\begin{tabular}{|c|c|c|c|c|c|c|}
\hline \multirow[t]{2}{*}{ MMSE } & \multicolumn{6}{|c|}{ Time post-onset of stroke } \\
\hline & 20 day & 36 day & 47 day & 60 day & 10 mon & 16 mon \\
\hline Orientation (Time) & 1 & 0 & 2 & 2 & 5 & 4 \\
\hline Orientation (Place) & 0 & 4 & 5 & 5 & 5 & 4 \\
\hline Registration & 0 & 3 & 3 & 3 & 3 & 3 \\
\hline Attention and calculation & 0 & 0 & 0 & 1 & 1 & 3 \\
\hline Recall & 0 & 0 & 0 & 0 & 2 & 2 \\
\hline Language & 0 & 8 & 8 & 8 & 8 & 8 \\
\hline Copying & 0 & 0 & 0 & 0 & 0 & 1 \\
\hline Total & 1 & 15 & 18 & 19 & 24 & 25 \\
\hline
\end{tabular}

K-MMSE, Korean Mini-Mental State Examination.

Table 2. Evaluation scores

\begin{tabular}{|c|c|c|c|}
\hline & \multicolumn{3}{|c|}{ Time post-onset of stroke } \\
\hline & 20 day & 37 day & 40 day \\
\hline$\overline{C D R}$ & 7 & - & 1 \\
\hline GDS & 3 & - & 4 \\
\hline Verbal IQ & - & 64 & - \\
\hline Performance IQ & - & 69 & - \\
\hline Total IQ & Below 60 & 65 & - \\
\hline SQ & 2 (social age 1 ) & - & - \\
\hline Class & Moderate MR & Mild MR & - \\
\hline AQ score & 26.6 & 73.6 & - \\
\hline MMT & $\mathrm{P}-\mathrm{F}-/ \mathrm{N}$ & $\mathrm{G} / \mathrm{N}$ & - \\
\hline FIM & 42 & 91 & - \\
\hline
\end{tabular}

CDR, clinical dementia rating; GDS, global deterioration scale; IQ, intelligence quotient; SQ, self-happiness quotient; MR, mental retardation; AQ, aphasia quotient; MMT, manual muscle testing; P, poor; F, fair; N, normal; G, good; FIM, functional independence measure.

\section{DISCUSSION}

AChAI results in limited sub-cortical lesions involving the medial temporal cortex, the posterior limb of the internal capsule, the posterior and medial parts of the lentiform nucleus, the body of the caudate nucleus and the periventricular white matter. AChAI typically presents with several neurological deficits including motor weakness; more severe deficiencies and lesion extension can be a source of aphasia and spatial neglect [2].

Contrary to the established clinical features of AChAI, this case evidenced persistent cognitive impairment despite rehabilitation therapy, with near complete and rapid motor recovery. The patient's infarction site was in the posterior limb of the left internal capsule, the cortical sites connecting inferiorly through the corticospinal and corticopontine tracts. Generally, infarction in the posterior limb of the internal capsule is classified as pure motor stroke. However, because this patient's motor function recovery was rapid, we performed DTI brain tractography to investigate the reason behind this rapid recovery [3].

DTI is a MRI technique that can be used to assess the structural integrity of the corticospinal tract (CST) and is generally used to determine the relationship between motor function and the CST. Nelles et al. [4] reported that unfavorable motor outcome of patients with a sole infarct in the AchA territory showed positive correlation with more notably decreased fractional anisotropy of diffusion tensor tractography. In this case, DTI brain tractography evidenced that both CSTs were intact, although there was infarction in the posterior limb of the left internal capsule. Rapid motor function recovery of this patient was based on these results. Additionally, it can be surmised that the other tracts involved in motor function such as the reticulospinal tract, vestibulospinal tract, and rubrospinal tract were intact as well $[5,6]$. 
The notable finding in this case was persistent cognitive impairment. Severe AChAI may represent the source of aphasia and spatial neglect, but there have been scarce reviews on other possible cognitive disorders. The patient's cognitive impairment persisted despite cognitive rehabilitation therapy. Although the language ability recovered faster than other cognitive function sectors, attention, calculation and memory were still significantly impaired 16 months following onset.

Sarangi et al. [7] reported a case of AChAI presenting progressive cognitive deficit that caused predominantly memory symptoms in an elderly. The patient had medical history about several years of depression and memory loss and cognitive abnormalities consistent with Alzheimer's disease (AD). The brain single-photon emission computed tomography (SPECT) and brain MRI were performed. As SPECT demonstrated the decreased cerebral blood flow on right parahippocampal cortex, following MRI confirmed the infarction on this region. However, there has been no progressive cognitive dysfunction in our case, rather it occurred abruptly. Thus, we did not consider $\mathrm{AD}$ or other disorders that cause progressive dementia at the onset. Indeed, the MR image did not reveal further ischemic lesions other than the territory that left AChA.

AChAI lesions involve the associative tracts joining the cortex and the thalamus. Maeshima et al. [8] reported a case of dysgraphia due to AChAI. In that case, although MRI revealed only infarction on the left subcortical region, SPECT additionally revealed decreased cerebral blood flow in the left frontal and parietal cortex. As AChA supplies the thalamus, lentiform nucleus, and dorsolateral hemispheric cortex, the authors insist that occlusion of this artery would disconnected the thalamocortical radiation. Likewise, we can assume that the cognitive impairment may be due to the damage of the thalamocortical tract which connects the posterior limb of the left internal capsule to the frontal and parietal cortex [9]. Additionally, there was a report about the pathways joining the cerebellum to the premotor cortex travel through the internal capsule, and lesions affecting these pathways could also contribute to cognitive deficits in attention, memory, and executive function [10].

Kwan et al. [11] demonstarated that subcortical infarction produces global cerebral hypometabolism with clinical relation by $18 \mathrm{~F}$-fludeoxyglucose positron emission tomography/computed tomography. Considering that the lesions involving the thalamocortical tract or spinocerebellar tract also cause the cognitive dysfunction by literature review, we assume that the internal capsule as an intersection of these two tract as a candidate for cognitive dysfunction.

The major limitation of our study is lack of functional imaging studies. DTI was insufficient to review the connectivities of the internal capsule with other anatomical lesions. Therefore, further imaging studies such as SPECT, positron emission tomography for detecting additional cortical hypoperfusion remote from the focal infarction site would be helpful to describe the unexpected cognitive dysfunction. Further, resting state functional MRI can be another tool for reveal the causes of this kind of unexpected symptoms after focal ischemic stroke in subcortical lesions [12].

In summary, acute infarction in the posterior limb of the left internal capsule supplied by the AChA could be the source of persistent cognitive impairment involving attention and memory. Since, it could contribute to difficulties in social and professional activities, demonstrating the underlying connectivities to remote lesions would be important to predict the prognosis and to make a short- and long-term goals of rehabilitation. 


\section{REFERENCES}

1. Pezzella FR, Vadalà R. Anterior choroidal artery territory infarction. Front Neurol Neurosci 2012;30:123-127. PUBMED | CROSSREF

2. Rousseaux M, Cabaret M, Serafi R, Kozlowski O. An evaluation of cognitive disorders after anterior choroidal artery infarction. J Neurol 2008;255:1405-1410.

PUBMED | CROSSREF

3. Jang SH, Kim K, Kim SH, Son SM, Jang WH, Kwon HG. The relation between motor function of stroke patients and diffusion tensor imaging findings for the corticospinal tract. Neurosci Lett 2014;572:1-6. PUBMED | CROSSREF

4. Nelles M, Gieseke J, Flacke S, Lachenmayer L, Schild HH, Urbach H. Diffusion tensor pyramidal tractography in patients with anterior choroidal artery infarcts. AJNR Am J Neuroradiol 2008;29:488-493. PUBMED | CROSSREF

5. Jankowska E, Edgley SA. How can corticospinal tract neurons contribute to ipsilateral movements? A question with implications for recovery of motor functions. Neuroscientist 2006;12:67-79. PUBMED | CROSSREF

6. Blumenfeld H. Neuroanatomy through Clinical Cases. 2nd ed. Sunderland, MA: Sinauer Associates; 2010. pp. 230-237.

7. Sarangi S, San Pedro EC, Mountz JM. Anterior choroidal artery infarction presenting as a progressive cognitive deficit. Clin Nucl Med 2000;25:187-190. PUBMED | CROSSREF

8. Maeshima S, Osawa A, Nagoya H, Takeda H, Tanahashi N. Dysgraphia due to anterior choroidal artery territory infarction: a case report. Neurol Sci 2013;34:1823-1825. PUBMED | CROSSREF

9. Rousseaux M, Froger J, Kozlowski O, Steinling M. [Cerebral blood flow disturbances after anterior choroidal artery infarcts. Anatomical and functional correlates]. Rev Neurol (Paris) 2001;157:187-197. PUBMED

10. Gottwald B, Wilde B, Mihajlovic Z, Mehdorn HM. Evidence for distinct cognitive deficits after focal cerebellar lesions. J Neurol Neurosurg Psychiatry 2004;75:1524-1531.

PUBMED | CROSSREF

11. Kwan LT, Reed BR, Eberling JL, Schuff N, Tanabe J, Norman D, et al. Effects of subcortical cerebral infarction on cortical glucose metabolism and cognitive function. Arch Neurol 1999;56:809-814. PUBMED | CROSSREF

12. Cabeza R, Nyberg L. Imaging cognition II: An empirical review of 275 PET and fMRI studies. J Cogn Neurosci 2000;12:1-47.

PUBMED | CROSSREF 